ORIGINAL ARTICLE

\title{
Patterns of Hepatocellular Carcinoma on Computed Tomography at Tikur Anbessa specialized hospital, Addis Ababa, Ethiopia
}

\section{Ferehiwot Getaneh $^{1 *}$, Asfaw Atnafu ${ }^{1}$}

\footnotetext{
OPEN ACCESS

Citation: Ferehiwot Getaneh, Asfaw Atnafu.. Patterns of Hepatocellular Carcinoma on Computed Tomography at Tikur Anbessa specialized hospital, Addis Ababa, Ethiopia. Ethiop J Health Sci.2020;30(2):209.

doi:http://dx.doi.org/10.4314/ejhs.v30 i2.8

Received: October 24, 2019

Accepted: November 22, 2019

Published: March 1, 2020

Copyright: (C2020 Ferehiwot Getaneh, et

al. This is an open access article distributed under the terms of the

Creative Commons Attribution License,

which permits unrestricted use,

distribution, and reproduction in any

medium, provided the original author and source are credited.

Funding: Nil

Competing Interests: The authors declare that this manuscript was approved by all authors in its form and that no competing interest exists.

Affiliation and Correspondence:

${ }^{1}$ Department of Radiology, College of

Health Sciences, Addis Ababa

University Addis Ababa, Ethiopia

*Email: teguazh@gmail.com
}

ABSTRACT

BACKGROUND: Hepatocellular cancer is the commonest liver cancer which contributes to a high incidence of morbidity and mortality in both developed and developing countries. Despite the anticipated high burden of the disease in the country, there is paucity of data on the associated risk factors and its pattern on imaging. The aim of this study was to assess Computed Tomography patterns and the risk factors of hepatocellular carcinoma.

METHODS: A cross-sectional, prospective study on patients with a diagnosis of HCC on Computed Tomography examination, at Tikur Anbessa Specialized Hospital from July, 2016 to July, 2017. All patients who had characteristic Computed Tomography features and those who were diagnosed by Fine needle aspiration cytology (FNAC)/biopsy and had Computed Tomography examination were included in the study. Risk factors identified were reviewed from patients' medical records.

RESULT: A total of 70(n=70) patients were included in the study. The mean age was $50 \pm 16$ years. Fifty-seven (81.4\%) of the participants were males, and 13(18.6\%) were females. Forty-five (64.3\%) of them came from urban areas and 25(35.7\%) of them were from rural areas. The lesions were focal in 52(74.3\%) and infiltrative in 18(25.7\%) of the cases. Vascular invasion and distant metastases were seen in $49(70 \%)$ and $17(24.3 \%)$ respectively. Twenty-nine (41\%) had negative hepatitis markers, and 13(19\%) were not investigated for hepatitis infection.

CONCLUSION: The majority of the patients presented with advanced disease such as vascular invasion and metastases. The major risk factors such as hepatitis infection were negative in significant number of patients. The risk factors in our setting should be studied further. Moreover, high-risk group selection and screening is essential to diagnose HCC early.

KEYWORDS: Computed Tomography, hepatocellular carcinoma, risk factors

\section{INTRODUCTION}

Hepatocellular carcinoma (HCC) is the commonest form of liver cancer, and most frequently (approximately 80\%), it is associated with chronic hepatitis B virus (HBV) or hepatitis C virus (HCV) 
Infections (1). Other non-viral causes such as metabolic syndromes, aflatoxin exposure, alcohol use, tobacco use, and oral contraceptive use are also risk factors for $\mathrm{HCC}$ (2). Most cases of $\mathrm{HCC}$ cases $(>80 \%)$ occur in sub-Saharan Africa and in Eastern Asia, with a typical incidence rates of $>20$ per 100,000 individuals (1).

There is no study on prevalence or incidence of hepatocellular cancer in Ethiopia. However, expected disease burden is significant because of high prevalence of hepatitis infection (approximately $10 \%-15 \%$ of $\mathrm{HBV}$ and that of $\mathrm{HCV}$ is approximately $2 \%-5 \%)(3)$.

By identifying the risk factors for $\mathrm{HCC}$, high risk groups can be selected and enrolled in screening programs. In fact, the management of high-risk patients with screening and surveillance allows early detection of HCC and improve patient outcomes. When HCC is detected earlier, patients are candidates to receive curative treatments (4).

Hepatic nodules can be diagnosed on Ultrasounds (US), including contrast-enhanced US (CEUS), or on other noninvasive techniques, such as contrast-enhanced computerized tomography (CT), magnetic resonance imaging (MRI) and positron emission tomography (PET)-CT. The typical feature of HCC on dynamic imaging is characterized by early arterial phase enhancement followed by loss of enhancement in the portal venous phase and delayed phase in comparison to the surrounding liver (5). In resource limited countries, patients come with advanced disease and there are only few treatment options for HCC. In our study, we assessed patterns of the disease on $\mathrm{CT}$ and if there were identifiable risk factors.

\section{METHODS}

The study was conducted at Tikur Anbessa Specialized Hospital, Addis Ababa, Ethiopia. It is a tertiary hospital in the country where the only cancer center is located, and patients from all over the regions are referred to for better diagnostic and therapeutic services. The CT scanners were GE (General Electric) 64 slice and Philips 128 slice CT machines. The image quality acquired by the two CT scanners were comparable. Pre-contrast, arterial phase, portal venous phase and delayed phase images were taken consecutively.

It is a cross-sectional prospective study which was carried out from July 2016 to July 2017 on consecutive patients who came for Computed Tomography examination to the Department of Radiology. Those who have CT characteristic feature of hepatocellular cancer or those who have CT with histopathology confirmation of hepatocellular cancer were included in the study.

CT findings were reviewed and filled in structured questionnaire by the principal investigator. The diagnosis of hepatocellular carcinoma can be done non-invasively on the basis of typical imaging feature (6). A late arterial enhancement and venous or delayed phase washout in the background of cirrhosis or in patients with chronic hepatitis B infections was considered typical feature of HCC. All other clinical information including documented risk factors were reviewed from patients' medical records.

Statistical analysis: The statistical analysis was performed using the Statistical Package for Social Sciences (SPSS) for windows version 20. Mean was calculated for continuous variables. Frequencies were used to summarize categorical variables. Fisher's exact test was used to assess the significance of association between independent and dependent variables. The level of significance was considered as $\mathrm{P}<0.05$.

Ethical consideration: Ethical approval to conduct the study was obtained from Ethics Review Committee of the Department of Radiology before the commencement of the study.

\section{RESULTS}

A total of $70(n=70)$ people were included in the study. Demographic characteristics of patients is shown in Table 1. The mean age was $50 \pm 16$ years. Fifty-seven $(81.4 \%)$ of the participants were males and $13(18.6 \%)$ were females. Forty-five (64.3\%) came from urban areas, and $25(35.7 \%)$ of them were from rural areas.

Table.1: Socio-demographic characteristics of patients diagnosed with HCC at Tikur Anbessa specialized Hospital of year 2016-2017.

\begin{tabular}{lll}
\hline \multicolumn{2}{l}{ Characteristics } & Number(Mean \pm SD) \\
\hline Age & $49.7 \pm 16.5$ \\
Address of respondents & \\
& Rural & 25 \\
& Urban & 45 \\
Sex & & \\
& Female & 13 \\
& Male & 57 \\
& Total & $\mathbf{7 0}$ \\
\hline
\end{tabular}

DOI: http://dx.doi.org/10.4314/ejhs.v30i2.8 
Table 2: CT imaging pattern of 70 patients with HCC at Tikur Anbessa specialized Hospital of the year 2016-2017. APE=arterial phase enhancement, $\mathrm{PVP}=$ portal venous phase and $\mathrm{DP}=$ delayed phase

\begin{tabular}{llll}
\hline Characteristics & \multicolumn{2}{l}{ Imaging pattern frequency (\%) } & \\
\hline Location & Right lobe 19(27\%) & Left lobe 9(13\%) & Both lobes 42(60\%) \\
Pre contrast density & Hypodense 52(74\%) & Hyperdense 1(1.4\%) & Heterogeneous 1(1.4\%) \\
Enhancement pattern & APE 70(100\%) & PVP washout 62(89\%) & DP washout 18(11\%) \\
Mass lesion & Focal 52(74.3\%) & Infiltrative 18(23.7) & \\
Vascular invasion & Yes 48(69\%) & No 22(31\%) & \\
Biliary invasion & Yes 5(7\%) & No 65(83\%) & \\
Metastasis & Yes 17(24\%) & No 53(76\%) & \\
Cirrhosis & Yes 44(63\%) & No 26(37\%) & \\
\hline
\end{tabular}

Patterns depicted on CT are presented in Table 2. In $42(60 \%)$ of the patients, both hepatic lobes were involved by the tumor, while in $19(27 \%)$ and $9(13 \%)$, it was localized to right lobe and left lobe respectively. Fifty-two $(74.3 \%)$ of the lesions were focal (Figure 1) while 18(23.7\%) were infiltrative (Figure 2).

Invasion of the vessels was demonstrated in $48(69 \%)$ of them; from which the portal vein invasion contributed in $39(55.7 \%)$ of the cases (Figure 3) (Table 2). Biliary invasion was observed in $5(7.1 \%)$.

Tumors' density in the pre-contrast images was hypo in 52(74.3\%), hyper and heterogeneous in $1(1.4 \%)$ cases each. Pre-contrast image was not available in $16(22.9 \%)$ of the cases. Late arterial enhancement and venous phase washout are seen in $62(88.6 \%)$ and late arterial enhancement and delayed phase washout in $8(11.4 \%)$.

Liver cirrhosis was identified in $44(62.9 \%)$ and no CT evidence of cirrhosis in $26(37.1 \%)$ of the cases. Forty-four (62.9\%) had ascites. Splenomegaly was seen in 20(28.6\%). Fifteen (21.4\%) had lymph node metastasis. No distant metastasis is seen in $53(75.7 \%)$ cases, and there was metastases to the lung and adrenal in 9(12.9\%) and $4(5.7 \%)$ respectively. Other site metastasis was detected in $3(4.3 \%) ; 1(1.4 \%)$ of the cases had lung, adrenal and bone metastases.

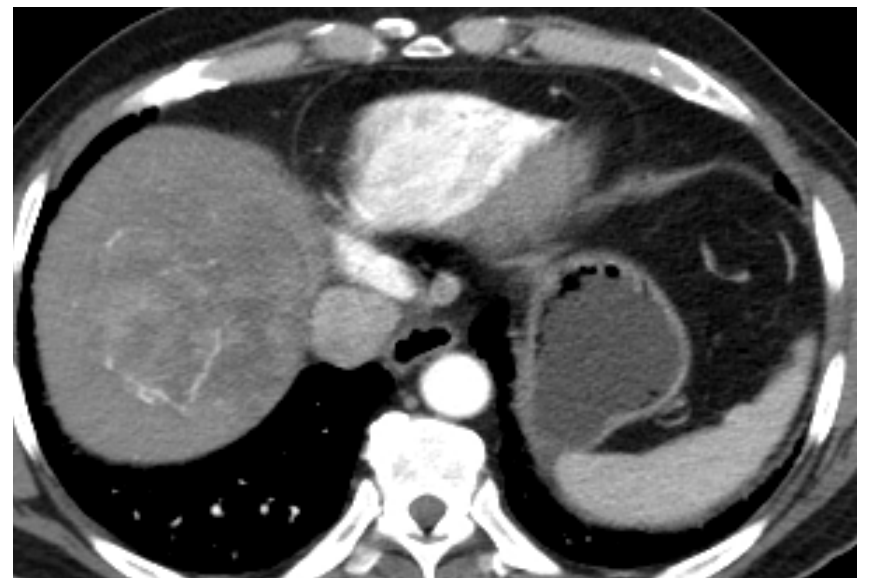

A

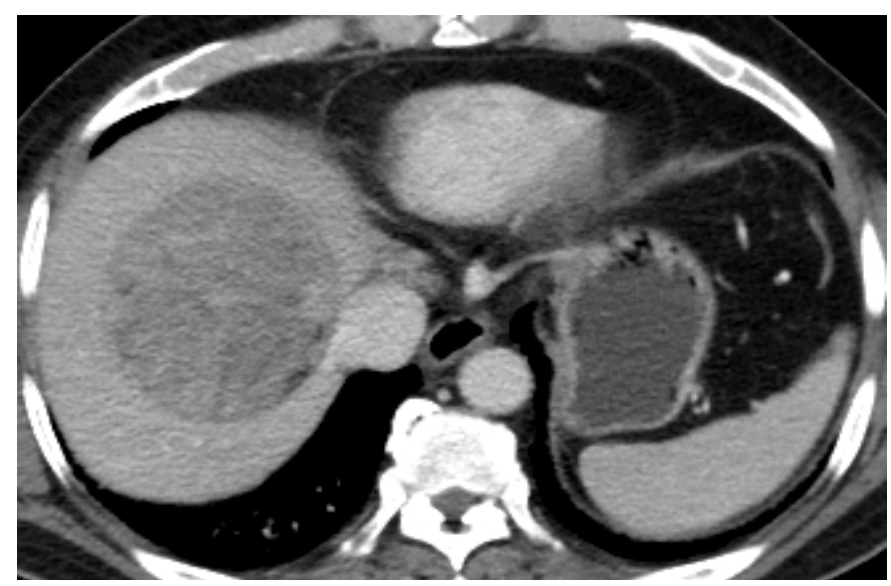

B

Figure 1: CT images of a 72 year old male patient with hepatocellular cancer A; well defined heterogeneously hyperenhancing lesion with intratumoral neovascularization in arterial phase B; washout of the lesion in the portal venous phase

DOI: http://dx.doi.org/10.4314/ejhs.v30i2.2 

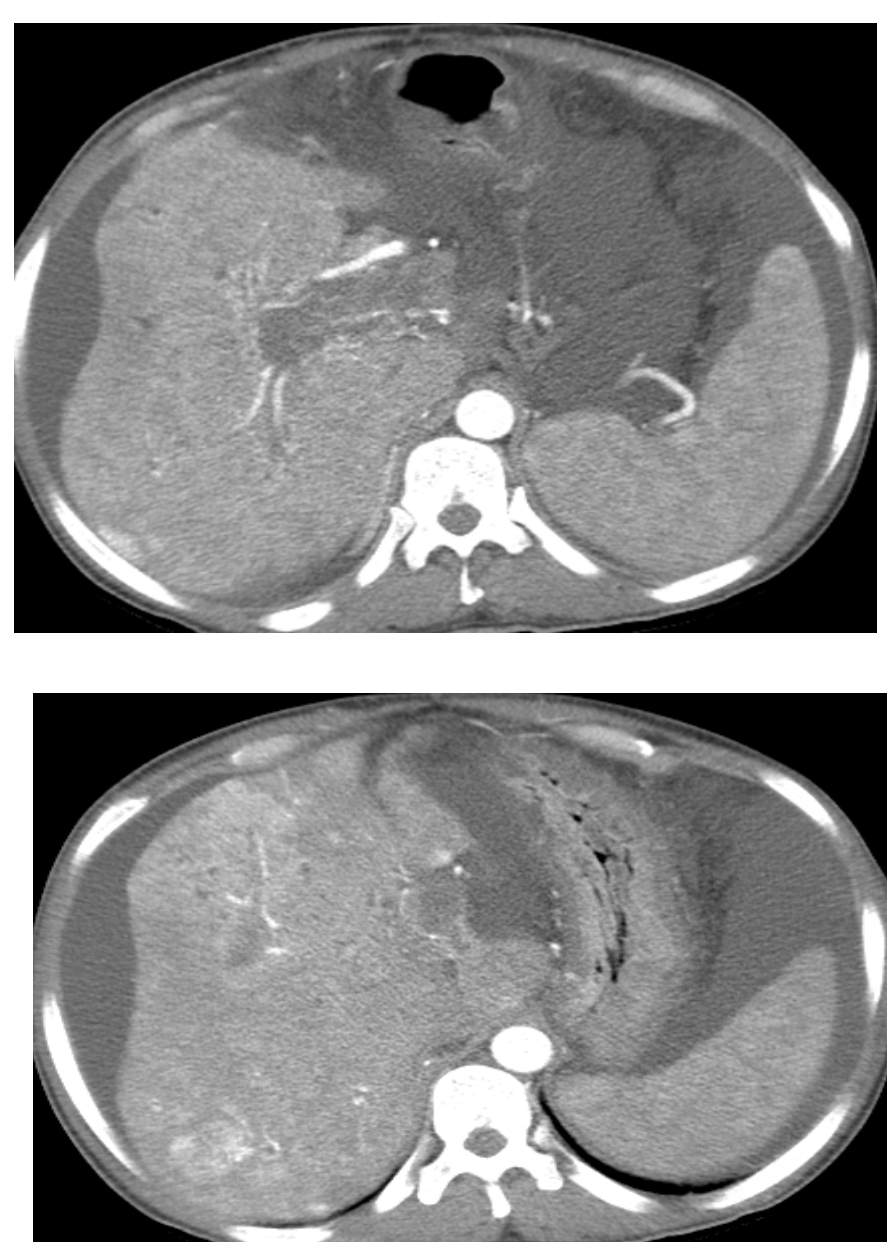

Table 3 shows risk factors identified. Positive HBV and $\mathrm{HCV}$ test were revealed in $15(21.4 \%)$ and $5(7.1 \%)$ respectively. Metabolic syndrome was a risk factor in $2(2.9 \%)$ and HIV in $1(1.4 \%)$. Other risk factors contributed to $4(5.7 \%)$ of the cases. In $1(1.4 \%)$ of the cases, concomitant HBV, HIV, HBV and other risk factors were identified. Hepatitis B surface antigen (HBsAg) and Hepatitis C virus

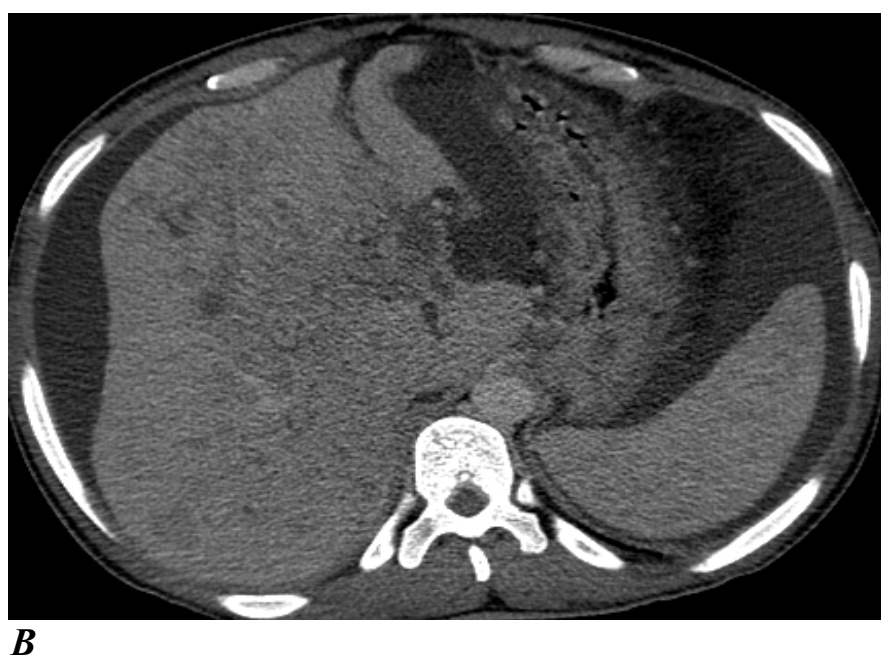

Figure 2: CT images of a 40 year old male patient with hepatocellular cancer A; An infiltrative and hyper-enhancing lesion on segment IVB of the liver on the arterial phase B. washout in the portal venous phase. $C$. Tumor thrombosis in the main and branches of the portal vein (arrows)

C

antibody (HCVab) results were negative in $29(41 \%)$ participants, and the tests were not performed in $19.1 \%$ of the patients. Overall, the major risk factors were not identified in $41(58.6 \%)$ of the cases. HCC was confirmed by histopathology in $12(17.1 \%)$ of the patients. Infiltrative lesions were more associated with vascular invasion than focal lesions $(\mathrm{P}=0.005)$.

Table 3: Pattern of risk factors identified in 70 HCC patients at Tikur Anbessa Hospital of year 2016-2017

\begin{tabular}{lll}
\hline Risk factor identified & Frequency & percent \\
\hline HBV & 15 & 21.4 \\
HCV & 5 & 7.1 \\
Metabolic syndrome & 2 & 2.9 \\
HIV & 1 & 1.4 \\
Unknown & 41 & 58.6 \\
Others & 4 & 5.7 \\
HBV and HIV & 1 & 1.4 \\
HBV and other & 1 & 1.4 \\
Total & $\mathbf{7 0}$ & $\mathbf{1 0 0}$ \\
\hline
\end{tabular}

DOI: http://dx.doi.org/10.4314/ejhs.v30i2.8 


\section{DISCUSSION}

The majority $(81.4 \%)$ of the participants in our study were males, with male-to female-ratio of $4: 1$. Similarly, higher male-to-female ratio of hepatocellular cancer averaging between $2: 1$ and $4: 1$ is reported in most population, and it is higher in high and medium incidence areas (7). Factors attributing to male predominance are hormonal effect (testosterone), the overall higher rate of cancer in male population, high exposure rate of HBV infection in males and others $(7,8)$. Mean age was $49.7 \pm 16$ yrs. The most common affected age groups are age $>65 \mathrm{yrs}$, which is $24.3 \%$ of the study subjects. This result has inconsistent pattern compared to a study done at Tikur Anbessa Hospital in which the age group of 55-64yrs accounted for $51 \%$ of the cases (9). We could not justify the difference between two results, and larger sample size study is suggested to understand overall demographic characteristics of $\mathrm{HCC}$ patients in the country (10).

In $23.7 \%$ of the cases, tumor is infiltrative which is slightly higher compared to similar study in Italy and lower compared to another study in Tanzania where they reported $20 \%$ and $26.6 \%$ respectively $(10,11)$. Those with infiltrative type of HCC also have signs of advanced disease such as vascular invasion. Portal invasion was seen in $55.7 \%$ of the cases. This is consistent with figures in literatures which state portal vein invasion in 44$62.8 \%$ of the times. Portal and/or other vascular invasions were seen in $70 \%$ of the cases (12). Lymph node metastasis and distant metastasis reported in $21.4 \%$ and $24 \%$ respectively. The lung was the most frequent $(53 \%)$ site of metastases. This result is in agreement with a study done in the University of Pittsburgh Medical Center where they found $55 \%$ of distant metastasis in the lung (13). Background liver cirrhosis is demonstrated in $62.9 \%$ of the cases. Liver cirrhosis was found in a relatively higher $(66.2 \%)$ of the cases in Indian study (11).

HBV and HCV markers were positive in $21.4 \%$ and $7.1 \%$ of the cases respectively. Hepatitis $\mathrm{B}$ surface antigen (HBsAg) and Hepatitis $\mathrm{C}$ virus antibody (HCVab) results were negative in 29(41\%) participants, and the tests were not performed in $13(19.1 \%)$ of the patients. In this study, relatively a comparable number of $\mathrm{HBV}$ infection and less number of $\mathrm{HCV}$ infection reported in a study undertaken in Ethiopia where both markers were positive in $24 \%$ of cases. Both $\mathrm{HBV}$ and $\mathrm{HCV}$ infections were less compared to the study in Tanzania where they found $65 \%$ and $16.9 \%$ of cases positive for $\mathrm{HBsAg}$ and $\mathrm{HCV}$ antibody respectively $(9,10)$. Risk factors assessment was difficult in this study due to incomplete laboratory workup and clinical data. More sensitive diagnostic tests such as HBV DNA and HCV RNA tests should be administered to detect occult hepatitis infections.

In conclusion, the majority of the patients in this study had evidence of advanced disease such as vascular invasion and metastasis at the time of presentation. A significant number of the patients had negative results for major risk factors. Further investigation is recommended regarding the risk factors of HCC in our setting; introduction of surveillance program for high-risk groups can improve early detection of the disease.

\section{REFERENCES}

1. El-Serag HB. Epidemiology of Viral Hepatitis and Hepatocellular Carcinoma. Gastroenterology. $\quad 2012 \quad$ 2012/05/01/; 142(6):1264-73.

2. Blonski W, Kotlyar DS, Forde KA. Non-viral causes of hepatocellular carcinoma. World $J$ Gastroenterol 2010; 16:3603-15.

3. Abate Bane, Aravind Patil, and Mahafroz Khatib. Healthcare cost and access to care for viral hepatitis in Ethiopia, Expert survey findings 2014. International Journal of Innovation and Applied Studies ISSN 20289324 Vol. 9 No. 4 Dec. 2014, pp. 1718-1723.

4. Francesco Bellissimo, Diagnostic and therapeutic management of hepatocellular carcinoma, World J Gastroenterol 2015 November 14; 21(42): 12003-12021.

5. Marrero JA, Hussain HK, Nghiem HV, Umar R, Fontana RJ, Lok AS. Improving the prediction of hepatocellular carcinoma in cirrhotic patients with an arterially-enhancing liver mass. Liver Transpl 2005; 11: 281-289. 
6. Leoni S, Piscaglia F, Golferi R, et al. The impact of vascular and nonvascular findings on the noninvasive diagnosis of small hepatocellular carcinoma based on the EASL and AASLD criteria. Am $J$ Gastroenterol 2010; 105: 599-609.

7. Helena Nordenstedt, Donna L. White, and Hashem B. El-Serag, the changing pattern of epidemiology in hepatocellular carcinoma, Dig Liver Dis. 2010; 42(Suppl 3): S206S214.

8. Yuan JM, Ross RK, Stanczyk FZ, et al. A cohort study of serum testosterone and hepatocellular carcinoma in Shanghai, China. Int J Cancer. 1995 Nov 15; 63(4):491-3.

9. Hailemichael Desalegn, Suraj Sharma, Abate Shewaye, Jordan Feld, Endale Lulu, Major risk factors, clinical and laboratory characteristics of patients with hepatocellular carcinoma; a retrospective study at Tikur Anbassa Hospital, Addis Ababa University, Addis Ababa, Ethiopia, Ethiop Med J, 2015, Vol. 53, No.3.
10. Hyasinta Jaka, Stephen E Mshana, Peter F Rambau, Nestory Masalu, Phillipo L Chalya and Samuel E Kalluvya, Hepatocellular carcinoma: clinicopathological profile and challenges of management in a resourcelimited setting, World Journal of Surgical Oncology 2014, 12:246.

11. Benvegnù L, Noventa $F$, Bernardinello E, Pontisso P, Gatta A, Alberti A. Evidence for an association between the aetiology of cirrhosis and pattern of hepatocellular carcinoma development. Gut 2001; 48(1):110-115.

12. Samir Shah, Akash Shukla, Bhawan Paunipagar, Radiological Features of Hepatocellular Carcinoma, $J$ CLIN EXP HEPATOL 2014; 4:S63-S66.

13. Sanjeev Katyal, James H. Oliver III, Mark S. Peterson, James V. Ferris, Brian S. Carr, Richard L. Baron, Extrahepatic Metastases of Hepatocellular Carcinoma, Radiology 2000; 216:698-703. 